\title{
Need of single target for single digit neonatal mortality and still birth rate
}

Keywords: neonatal, deaths, health, neonatal mortality, congenital malformations

Abbreviations: MDGs, millennium development goals; U5MR, under-five mortality; DNAP, district newborn action plan; HNAP, Haryana newborn action plan; INAP, newborn action plan; SDG, sustainable development goals; SBR, single digit stillbirth rate

\section{Opinion}

India was a signatory to the Millennium Declaration 2000 and has consistently reaffirmed its commitment towards the Millennium development goals (MDGs'). We have shown great progress in the MDGs' era, but narrowly missed its MDG 4 target for under-five mortality (U5MR), achieving a U5MR of 43 against the target of 42 per 1000 live births. ${ }^{1}$ Although India accounts for the highest number of under-5 deaths, it has shown a faster decline in U5MR compared to global statistics.

India's U5MR declined from 125 per 1,000 live births in 1990 to 43 per 1,000 live births in 2015 (reduced by $66 \%$ ) compared to the global rate of 90 per 1000 live births in 1990 to 42 per 1000 live births in 2015 (reduced by 54\%). Similarly, infant mortality rate also decreased from 68 per 1000 live births in 2000 to 38 per 1000 live births in 2015 (44\% reduction) and NMR from 40 per 1000 live births in 2000 to 25 per 1000 live births in 2015 (38\% reduction). ${ }^{2}$ Out of all three, the NMR has shown least impressive fall. NMR accounts for two-thirds of IMR and $60 \%$ of U5MR.

India has the highest number of neonatal deaths in the world recording 760000 neonatal deaths every year. In order to accelerate the fall in NMR, it is important to prioritize the neonatal health in the country. The major causes of newborn deaths in India are prematurity (35\%); neonatal infections (33\%); perinatal asphyxia (20\%); and congenital malformations ( $9 \%)$.

Most of the neonatal deaths are preventable by improving antenatal care, quality of care during delivery and care at birth. India has taken many steps in this direction and is committed to ending preventable newborn deaths to achieve "single digit NMR" by 2030, with all the states to individually achieve this target by $2035 .{ }^{3}$ However, only few states like Haryana have state newborn action plan (Haryana Newborn Action Plan) and district newborn action plan (DNAP) in place.

In the last three years, major commitments towards neonatal health are India Newborn Action Plan (INAP), Sustainable Development Goals (SDG) and National health policy $2017 \cdot \cdot^{3-5}$ All three have a broader goal of decreasing NMR but have varying targets. INAP is committed to achieving "single digit NMR" by 2030, SDG targets to reduce NMR to 12 by 2030 and National health policy 2017 to reduce neonatal mortality to 16 by 2025 .

\author{
Volume 2 Issue 4 - 2018
}

Jogender Kumar

Postgraduate Institute of Medical Education and Research, India

Correspondence: Jogender Kumar, Postgraduate Institute of Medical Education and Research, \#I- I35, MDH, Sector I2, Chandigarh, India, Tel 708765337,

Email jogendrayadv@gmail.com

Received: June 05, 2018 | Published: July 06, 2018

Similarly; for stillbirths INAP targets to achieve "single digit stillbirth rate (SBR)" by 2030 which is 5years ahead of Global every newborn action plan, however, our national health policy kept its target of single-digit SBR 5years ahead of INAP. SDG didn't commit anything about stillbirths.

For a country of unity in diversity like India, there is a need to have single well-defined targets with all efforts starting from district level directed towards it. Integration and convergence which are the basic principle of achieving success are lost due to lack of uniformity in our targets. With the given figure we might be succeeding in achieving the goal of INAP but will be failing with national health policy. So, do we need to celebrate it as the success or cry over the lost game?

\section{Acknowledgments}

None.

\section{Conflict of interest}

Author declares no conflict of interest.

\section{References}

1. United Nations Development Programme, India. India and the MDGs. 2015.

2. UNICEF. Child survival current status and progress.

3. Ministry of Health \& Family Welfare. Government of India. INAP: India Newborn Action Plan. 2014.

4. United Nations Development Programme, India. A New Sustainable Development Agenda. 2015.

5. Ministry of Health and Family Welfare, Government of India. National Health Policy 2017. New Delhi: MoHFW; 2017. 31 p. 